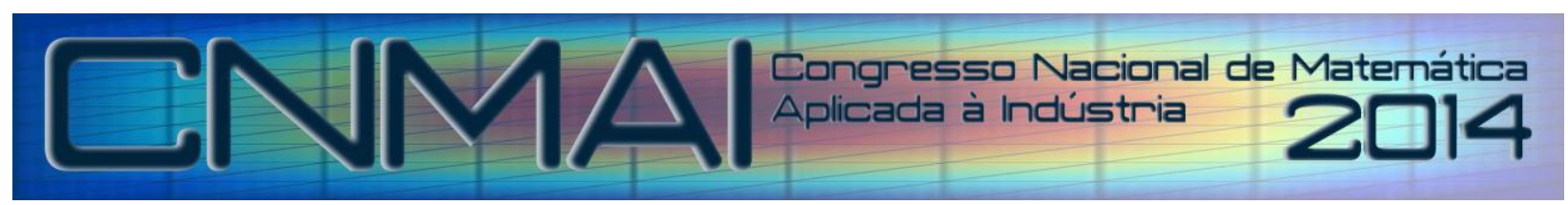

18 a 21 de novembro de 2014, Caldas Novas - Goiás

\title{
PROTÓTIPO DE ESTACIONAMENTO AUTOMATIZADO UTILIZANDO MODELO COMPUTACIONAL MATRICIAL E MICROCONTROLADOR ARDUINO
}

\author{
Thayanne Barros Bandeira, thayane.bandeira@ hotmail.com ${ }^{1}$ \\ Wellington Fonseca, Fonseca@ ufpa.br ${ }^{2}$ \\ Filipe Cavalcanti Fernandes, 13filipe11@ gmail.com ${ }^{3}$ \\ Alberto Chéquer Novaes Neto, alberto.chequer@gmail.com ${ }^{4}$
}

\begin{abstract}
${ }^{1}$ Universidade Federal do Pará - Campus Universitário de Tucuruí - Faculdade de Engenharia Elétrica - Rodovia BR
22 Km 13 - Canteiro de Obras - UHE Tucuruí - Vila Permanente 68464-000 - Tucuruí - Pará

${ }^{2}$ Universidade Federal do Pará - Campus Universitário de Tucuruí - Faculdade de Engenharia Elétrica - Rodovia BR

22 Km 13 - Canteiro de Obras - UHE Tucuruí - Vila Permanente 68464-000 - Tucuruí - Pará

${ }^{3}$ Universidade Federal do Pará - Campus Universitário de Tucuruí - Faculdade de Engenharia Elétrica - Rodovia BR

22 Km 13 - Canteiro de Obras - UHE Tucuruí - Vila Permanente 68464-000 - Tucuruí - Pará

${ }^{4}$ Universidade Federal do Pará - Campus Universitário de Tucuruí - Faculdade de Engenharia da ComputaçãoRodovia BR 22 Km 13 - Canteiro de Obras - UHE Tucuruí - Vila Permanente 68464-000 - Tucuruí - Pará
\end{abstract}

Resumo: Uma realidade presente no cotidiano da população de grandes cidades é o aumento da frota de veículos, que a cada dia se torna mais expressivo a medida que esses grandes centros urbanos crescem e o mercado automobilístico é estimulado. Esse dilema é diretamente relacionado com a dificuldade dos motoristas em encontrar vagas disponíveis em estacionamentos, tanto públicos quanto privados. A vista disso, iniciativa privada de empresas estrangeiras se atentam para implementar sistemas complexos e de custo elevado de controle e automação para gerenciamento de estacionamentos. Tendo em mente essa abordagem, o presente artigo propõe o desenvolvimento de um sistema de controle integrando conceitos de eletrônica e computação, capaz de operar e gerenciar através de procedimentos automatizados estacionamentos de médio e grande porte. Esse sistema tem seu princípio de funcionamento o modelo computacional matricial. O projeto utiliza a plataforma open source Arduino para localizar e contabilizar as vagas disponíveis por meio de sensores ultrassônicos e LDR que detectam a presença do veículo. Na sequencia, através de um sistema de comunicação em que as vagas compartilham a mesma conexão e cada uma é identificada por meio de um número binário, o microcontrolador é responsável por enviar aos displays localizados ao longo da trajetória dos veículos, o número total de vagas não ocupadas, referente tanto ao público em geral quanto para idosos e portadores de necessidades especiais. Portanto, o objetivo deste trabalho é desenvolver um método eficaz para circulação de automóveis dentro desses estabelecimentos principalmente em horários de grande fluxo.

Palavras-chave: Estacionamento, Automação, Arduino.

\section{INTRODUÇÃ̃O}

Os grandes centros urbanos estão em crescente ascensão nos dias atuais. A necessidade de se deslocar nessas grandes cidades, contribui para a expansão do número de veículos. Segundo a estatística do DENATRAN, Departamento Nacional de Trânsito, no final de 2013 a frota brasileira ultrapassou a casa dos 44 milhões de veículos, representando um crescimento de $46 \%$ em dez anos. Com isso, vários transtornos surgem na rotina diária, além do trânsito caótico e engarrafamentos, esse dilema é diretamente relacionado com a dificuldade dos motoristas em encontrar vagas disponíveis em estacionamentos.

A dificuldade em estacionar é presente dentro de estacionamentos públicos e privados devido à demora em encontrar vaga disponível. Esse problema tem se tornado questão mundial, inúmeras empresas se atentam para implementar sistemas de controle e automação para realizar este gerenciamento. Um bom estacionamento é a porta da frente, a primeira recepção aos clientes (CHAVES, 2010). 
Diante do problema de estacionar, possíveis soluções surgem cada vez mais inovadoras. Em meio a diversas soluções o conceito de estacionamento inteligente vem incorporando o ramo com recursos tecnológicos e caritativos que combinam o conforto dos usuários e o avanço científicos no mercado.

O Sistema Inteligente para Estacionamentos são soluções tecnológicas para o problema de fluxo no ato de estacionar (PSA, 2012). Sublimemente desenvolvido pela busca de inovações visando resultados que possam ser implementados para facilitar o trânsito aos usuários e ajudar os administradores do local, pois com a automação desses ambientes é possível coordenar o curso e a circulação de veículos fornecendo uma completa informação gerencial para o controle eficiente do uso das vagas proporcionando conforto e qualidade no serviço (BRAMITEC, 2013)

Tendo em mente essa abordagem, o presente artigo propõe o desenvolvimento de um sistema de controle e gerenciamento de vagas cobertas e descobertas que estejam desocupadas, de forma inovadora, econômica e ecologicamente correta. Integrando conceitos de eletrônica e computação, utilizando detecção inteligente de vagas e sinalização do ambiente para conduzir o motorista, o sistema é capaz de operar e organizar através de procedimentos automatizados estacionamentos de médio e grande porte.

O princípio de funcionamento do protótipo é um modelo computacional matricial. O projeto utiliza a plataforma open source Arduino para localizar e contabilizar as vagas disponíveis, através de uma rede de sensores instalados em cada vaga: ultrassônicos e resistor dependente de luz que detectam a presença do veículo. Em seguida, através de um sistema de comunicação, as vagas são identificadas por meio de um número binário. O microcontrolador é responsável por comunicar aos sinalizadores luminosos e displays estrategicamente posicionados ao longo da trajetória dos veículos. As informações em tempo real do número total de vagas não ocupadas são indicadas por LEDS (Diodo Emissor de Luz) de cores variadas, referente ao público em geral e especial: idosos e portadores de necessidades especiais.

Por tanto, o objetivo deste trabalho é desenvolver um método eficaz para gerir a circulação de automóveis a procura de vagas dentro desses estabelecimentos, poupando tempo, diminuindo o estresse dos condutores e reduzindo a emissão de gás Carbônico $\left(\mathrm{CO}^{2}\right)$ principalmente em horários de grande fluxo.

\section{METODOLOGIA}

A metodologia utilizada neste projeto consta na composição de diversas etapas, as quais foram testadas e implementadas de forma gradual para melhor análise de possíveis erros e melhor organização. Para implementação do mesmo foi necessário a análise dos componentes que farão o gerenciamento e correto funcionamento do protótipo de estacionamento automatizado, este projeto está fundamentado em dois elementos: Sistemas Automáticos e Plataforma de Prototipagem Eletrônica Arduino.

\subsection{Sistemas Automáticos}

A automação é considerada qualquer sistema apoiado em computadores que vise substituir tarefas de trabalho humano e/ou que vise soluções rápidas e econômicas para as indústrias e os serviços modernos (Castrucci \& Bottura, 2006).

Um sistema se caracteriza por um conjunto de elementos inter-relacionados, onde um comportamento de cada um deles afeta o comportamento dos outros elementos e do sistema como um todo. Dependendo do comportamento no sistema, nosso caso um estacionamento, uma determinada ação deve ser realizada. Esse comportamento denomina-se resposta do sistema, essa resposta está caracterizada por uma grandeza física que pode ser medida. Em nosso sistema serão medidas as condições do ambiente e conforme as mudanças neste ambiente, a devida ação será realizada, que no nosso caso o estacionamento mostrará quais vagas estarão disponíveis graças as leituras dos sensores nestes locais provendo devida sinalização aos motoristas sobre estes lugares. A figura 1 a seguir demonstra o diagrama de um sistema automatizado:

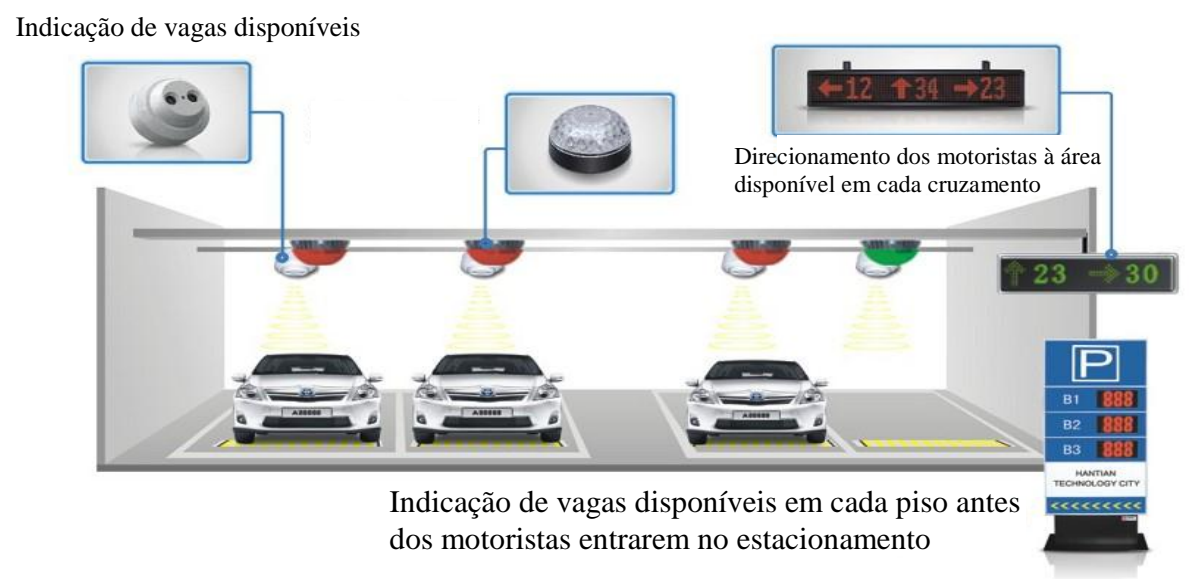

Figura 1. Diagrama de sistema automatizado. 


\subsection{Plataforma de Prototipagem Arduíno}

O Arduíno é uma plataforma de prototipagem eletrônica baseada na flexibilidade e fácil utilização entre software e hardware de código-aberto. A placa micro controladora foi lançada na Itália em 2005 e foi projetada com base nos microcontroladores da Atmel, ele possui 20 pinos que podem ser configurados tanto de entrada quanto saída (dos quais 6 podem ser usados como saída PWM - Modulação de Pulso de Largura), 6 entradas analógicas. Em sua estrutura possui um oscilador de cristal de $16 \mathrm{Mhz}$, uma porta USB, uma tomada de alimentação, um conector ICSP (Programação Serial de Circuito Interno), e um botão de reset. Para sua utilização basta conectá-lo a um computador com um cabo USB (Porta Serial Universal) ou liga-lo com um adaptador de Corrente Alternada para Corrente Contínua ou bateria (ARDUINO, 2012). Existem várias plataformas de Arduíno, o Arduíno uno foi o escolhido principalmente pela ótima aliança entre custo e benefício, além de ser apropriado ao projeto. O grande diferencial desta placa micro controladora também se deve a uma gama de possibilidades de interação com hardwares externos, no nosso caso sensores, LEDS e circuitos integrados. O Arduíno possui uma própria IDE (Ambiente de Desenvolvimento Integrado), onde a linguagem de programação padrão é $\mathrm{C} / \mathrm{C}++$ e funciona em várias plataformas computacionais como Windows, Linux e Mac OS, o dispositivo é ideal para criação de diversos ambientes interativos. A figura 2 a seguir apresenta a plataforma de prototipagem Arduino modelo Uno utilizada no protótipo:

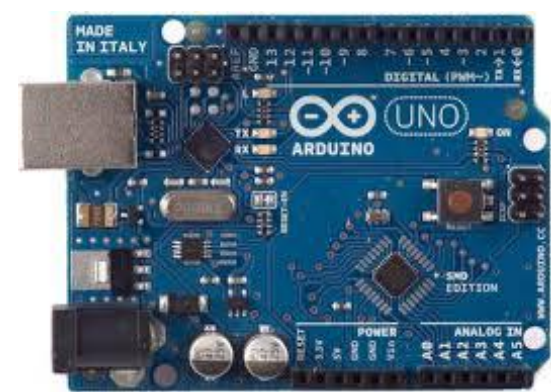

Figura 2. Plataforma Arduino, modelo Uno.

\section{MONTEGEM}

Após a avaliação dos conceitos básicos que fundamentam o projeto piloto, fez-se necessário organizar os equipamentos, montar e configurar o protótipo em questão. O protótipo piloto em andamento simula o sistema de gerenciamento de vagas em um estacionamento automatizado que informam de maneira inteligente a quantidade de vagas disponíveis detectadas pelos sensores estrategicamente posicionados, em seguida enviam os dados coletados ao Arduíno, na sequência transferem o número de vagas livres a dois displays de sete segmentos posicionados ao longo da trajetória dos carros. O protótipo funciona utilizando uma estrutura modelo construída com os componentes eletrônicos e materiais listados abaixo:

- Fios jumper;

- 1 Arduíno modelo Uno;

- 2 Displays de 7 segmentos;

- 3 Resistências 100 ohms;

- 1 circuito integrado 7404;

- 2 circuitos integrados CD4511BE ;

- 8 LEDS (Diodo Emissor de Luz);

- 4 Tubos de antena de TV;

- 3 Sensores LDR (Resistor Dependente de Luz);

- 1 Sensor Ultrassônico;

- 1 Compensado $50 \mathrm{~cm}^{2}$;

- 1 Isopor;

- 4 Hastes de Ferro;

- 1 chapa de Alumínio.

\subsection{Circuito}

O circuito é alimentado por um cabo USB conectado ao microcontrolador. Nos pinos digitais do Arduino todos os $L E D S$, sensores e os displays de 7 seguimentos encontram-se interligados, estes ocupam 12 pinos digitais e 5 pinos analógicos. Na Protoboard encontra-se 2 circuitos integrados CD4511BE conectados aos displays e 1 circuito integrado 7404 conectado aos $L E D S$.

O circuito funciona da seguinte maneira, conforme o algoritmo implementado no Arduino, os sensores reagem a luz (LDR) e ao cálculo de distância (Ultrassônico). O microcontrolador faz a leitura dos sensores e analisa a quantidade de vagas. Cada vaga possui um valor unitário, no Arduíno é transformado em um valor binário de 4 bits e é enviado de 
forma paralela ao Circuito Integrado CD4511BE que converte os valores para os displays de 7 seguimentos. No momento em que uma vaga é ocupada, o Arduino cessa o envio de sinal para o $L E D S$ verde correspondente que está ligado ao circuito integrado 7404 que inverte o sinal recebido, em consequência, o LEDS vermelho irá acender sinalizando que uma vaga foi ocupada. A figura 3 a seguir representa o circuito utilizado no protótipo:

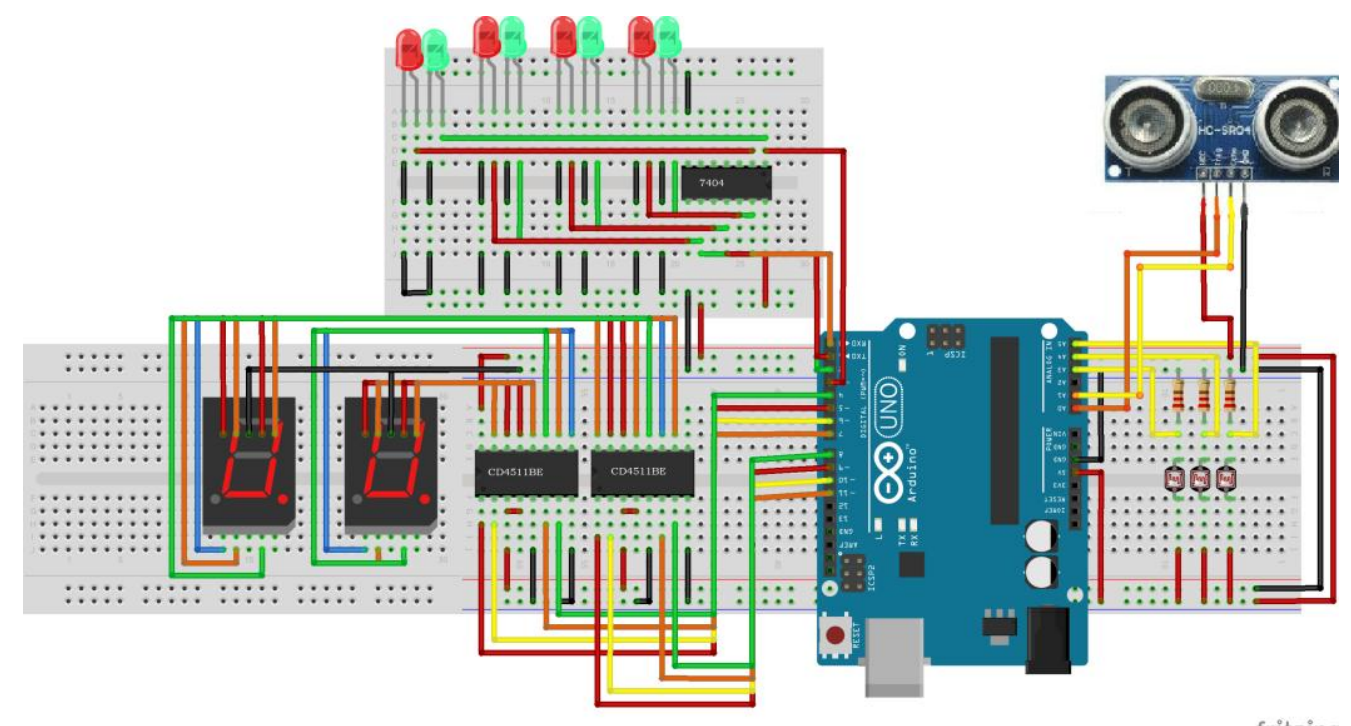

Figura 3: Circuito utilizado no protótipo.

\subsection{Protótipo de Estacionamento}

O protótipo utilizado neste trabalho teve sua construção inteiramente com materiais econômicos e de fácil montagem. Na representação da maquete foram utilizados um quadrado de $50 \mathrm{~cm}^{2}$ utilizado como base, um isopor cortado em pequenas partes e colocados nas extremidades do compensado que simbolizam as paredes do estacionamento, um pequeno retângulo de isopor foi utilizado como suporte para os dois displays de 7 seguimentos que representam a placa de informação das vagas disponíveis, as hastes de ferro servem de base para o telhado feito com uma chapa de alumínio, 4 tubos de uma antena de TV representando os postes no interior deles os fios foram conectados e estendidos ao Arduino. Os leds e os sensores foram fixados nos tubos de maneira estratégica, e estes foram interligados ao arduino. A Figura 4 (a), (b) e (c) apresenta o projeto pronto:

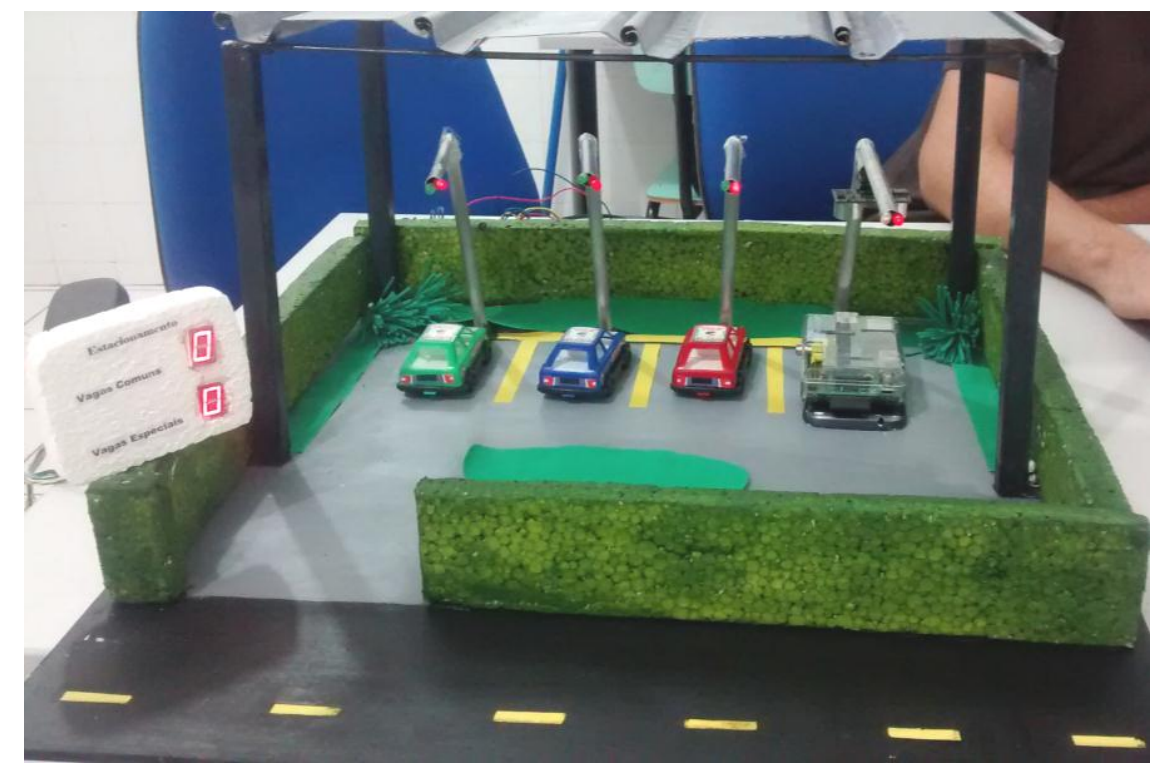

(a) Representa o protótipo em funcionamento, com todas as vagas ocupadas. 


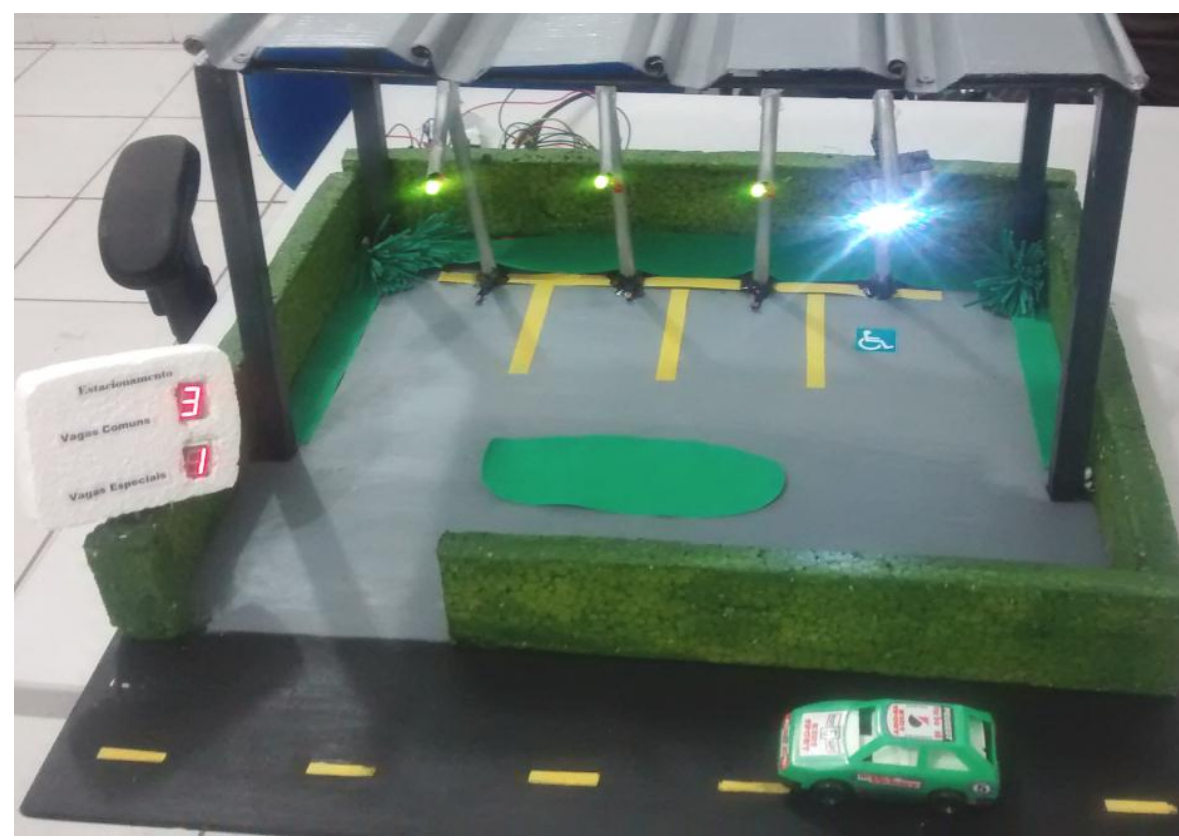

(b) Representa o protótipo em funcionamento, com todas as vagas desocupadas.

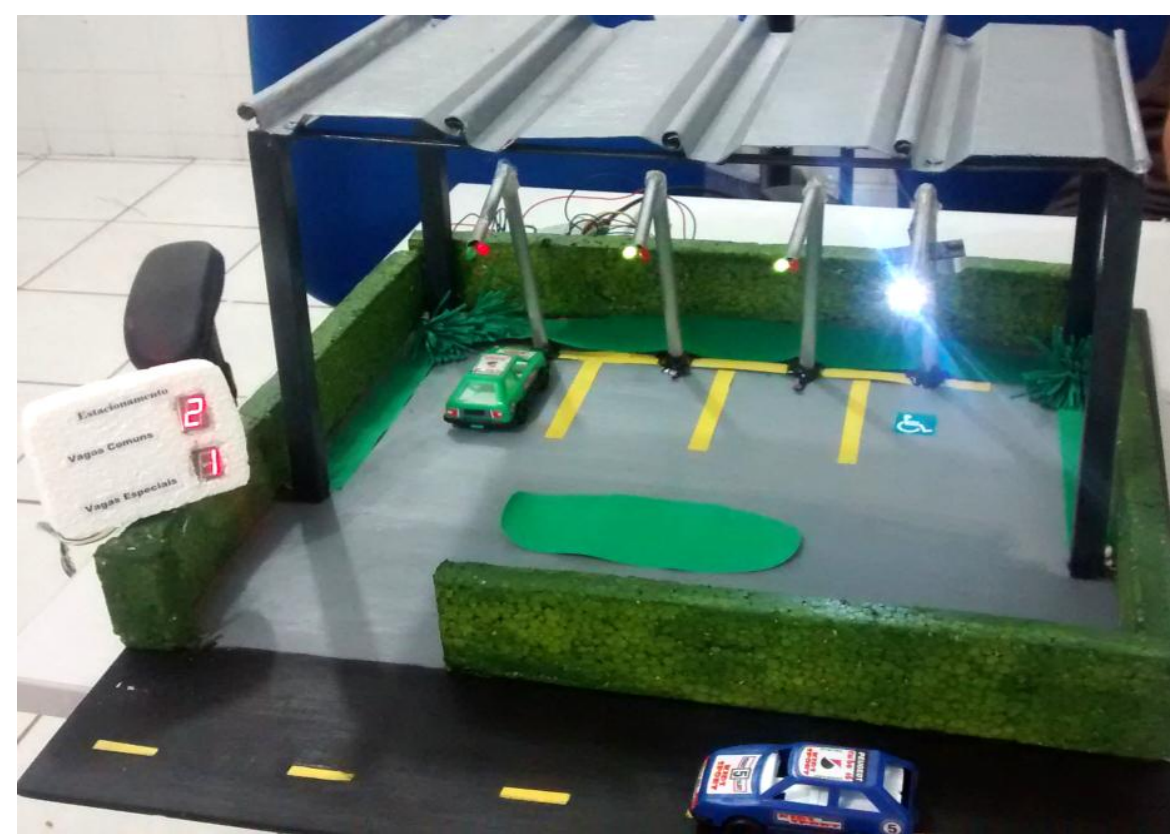

(c) Protótipo em funcionamento, $L E D S$ verdes representam vagas livres e vermelhos vagas ocupadas, no display o quantitativo de vagas.

Figura 4: Representação completa do funcionamento do protótipo.

\subsection{Algoritmo Implementado}

A lógica computacional representada na Figura 5 foi realizada através da IDE do próprio Arduíno. O micro controlador vai fazer a leitura dos sensores, processá-los e convertê-los em número binário que através dos circuitos integrados acionarão os $L E D S$ de notificação da vaga através dos seguintes passos:

- O microcontrolador inicia o programa, definindo as variáveis, constantes e os pinos respectivos aos sensores e $L E D S$.

- A leitura dos sensores é feita, possibilitando assim o controle dos $L E D S$ e a contagem das vagas não ocupadas que utilizam vetores para a identificação e sinalização.

- $\quad$ Feita a contagem, o resultado é colocado dentro de uma função que irá converter o mesmo para um número binário de 4 bits e enviado para o circuito integrado. 


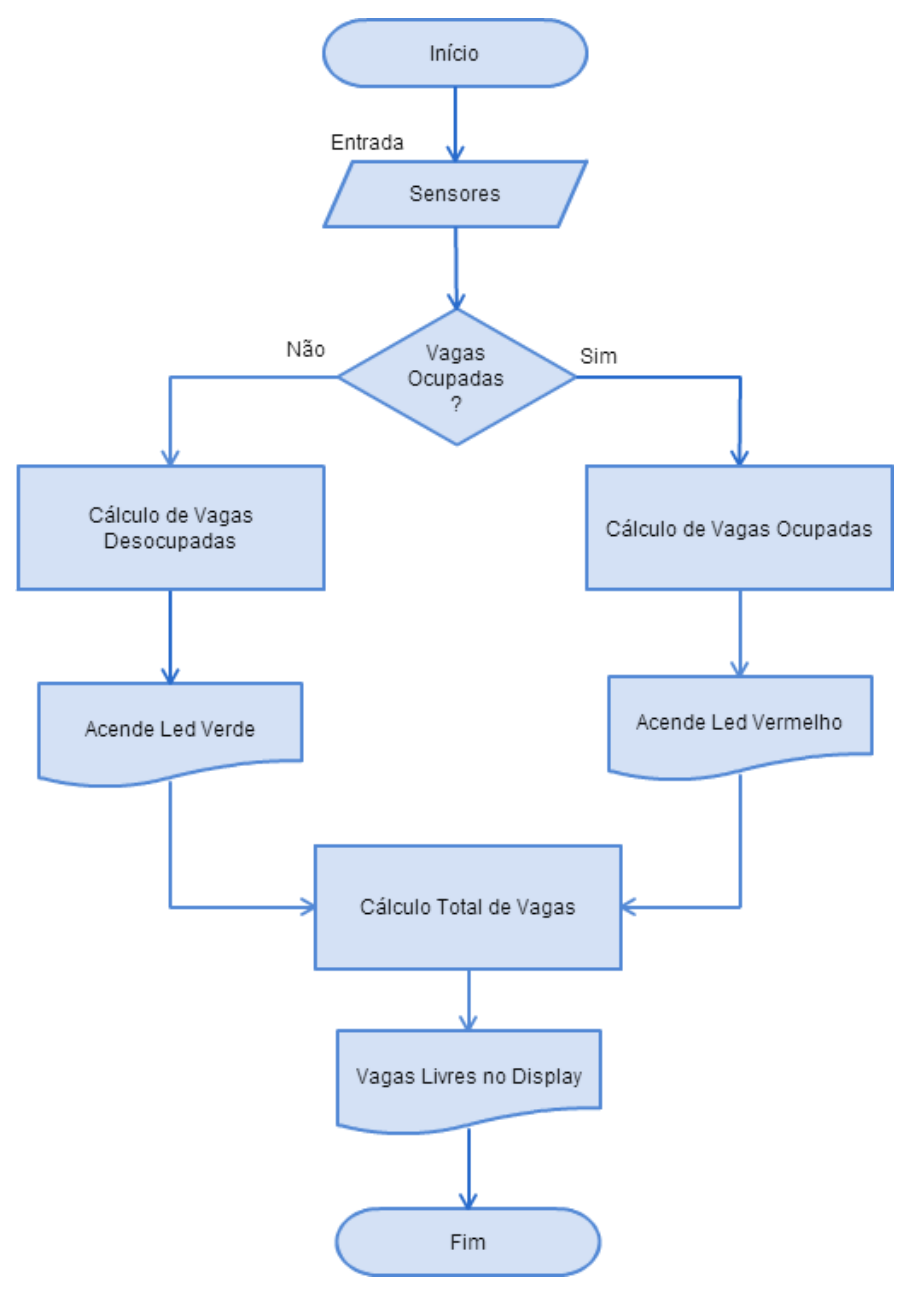

Figura 5: Diagrama do algoritmo utilizado no protótipo.

\section{RESULTADOS}

O sistema de detecção de vagas em estacionamento foi implementado utilizando a plataforma de prototipagem Aduíno. Foram feitos testes em ambiente simulado utilizando uma estrutura modelo, desenvolvida em formato de uma maquete que satisfaz diversos modelos de estacionamentos existentes e de tamanhos indefinidos. A figura 4 (a), (b) e (c) representam também alguns resultados do sistema algoritmo e circuito, ou seja, do software e hardware do protótipo.

$\mathrm{Na}$ figura citada acima, é possível notar o sistema proposto funcionando em três situações. Na figura 4 (a) simula a ação do sistema quando todas as vagas estão ocupadas. Na figura 4 (b) apresenta a simulação do sistema em condições em que nenhuma vaga está sendo ocupada. Na figura 4 (c) representa uma situação singular, em que apenas uma determinada vaga está sendo ocupada. É possível notar que conforme a quantidade de vagas ocupadas o número de vagas livres é disposto nos displays localizados na estrada do estacionamento. No entanto, segundo a situação da vaga um sinal luminoso é acionado, para o estado livre o sinal verde, para o estado ocupado o sinal vermelho e vagas especiais possuem cores diferentes, azul para livre e vermelho para ocupado.

Entretanto é importante ressaltar que o sistema foi testado inúmeras vezes de forma segura e confiável. O percentual de erros encontrados no sistema como um todo são relativamente pequenos, tornando possível e aplicável. O gráfico 1 a seguir apresenta o quantitativo de testes e erros no algoritmo e no circuito. 
Gráfico 1. Porcentagem de erro por seções de testes.

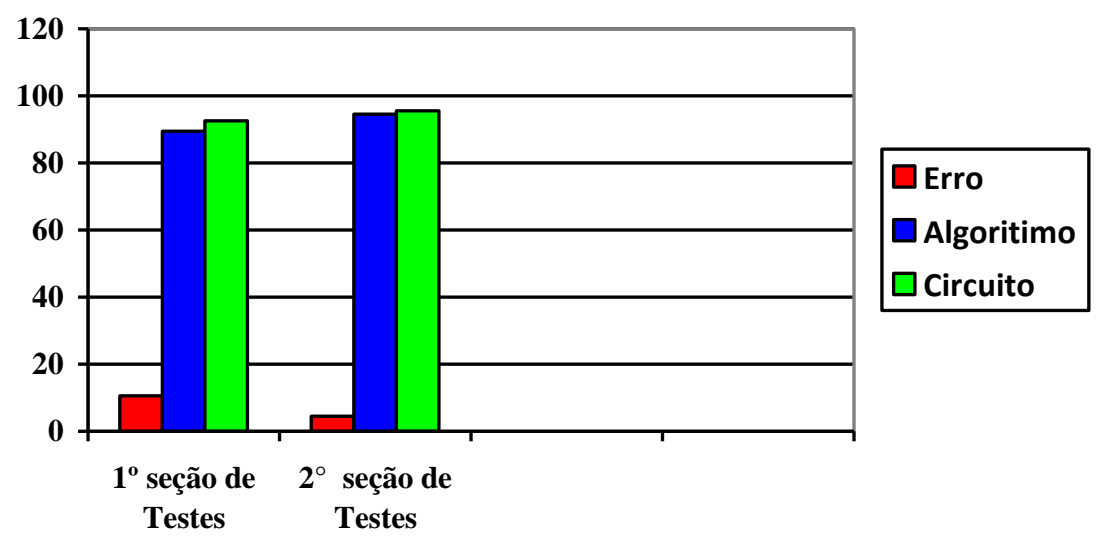

\title{
5. CONSIDERAÇÕES FINAIS
}

Atualmente existem muitos transtornos quanto à questão de estacionar em estacionamentos públicos e privados devido à demora em encontrar vaga disponível nos grandes centros urbanos que estão em ascensão nos dias atuais, soluções plausíveis surgem cada vez mais modernas para amenizar este fato, como os sistemas inteligentes para estacionamentos que permitem organizar fluxo no ato de estacionar.

Este trabalho contribuiu, trazendo uma visão para solucionar o problema de gerenciamento de estacionamentos autônomos, atuando em dois tipos de vagas: coberta e descoberta, utilizando dois tipos de sensores diferentes. Várias abordagens tratam da tarefa de automatizar estacionamentos, tanto no campo acadêmico como na indústria, no entanto ainda não validaram uma abordagem que consiga atuar deforma unificada nos diversos ambientes, o que existe são sistemas que atuam de forma específica.

Realizaram-se diversos testes experimentais utilizando uma estrutura modelo composta de um hardware e software desenvolvido para o sistema e os valores positivos atingidos com o sistema são bastante significativos como podemos visualizar no gráfico 1.

O protótipo desenvolvido atingiu todos os objetivos descritos inicialmente no artigo, de modo a conciliar conceitos eletrônicos e computacionais a fim de automatizar um estacionamento garantindo o conforto e a agilidade no fluxo de motoristas.

\section{REFERÊNCIAS}

Arduino. 2012. Overview. Disponível em: <http://arduino.cc/en/Main/ArduinoBoardUno> acessado em: 03 Mai. 2014;

Bramitec A. 2013. Sistemas de administração, monitoramento e orientação inteligente. São Paulo, v.1, n.1, p. 3-11, 2013;

Chaves, G. C. 2010. Estacionamento - um negócio da China! (e do Brasil, dos EUA, da Índia...). G9 Investimentos disponível em: <http://g9investimentos.com.br/artigos/ler/75.html> acessado em: 10 Mai. 2014;

DENATRAN. Departamento Nacional de Transito. 2013. Estatística anual de frota de veículos no Brasil. Disponível em: < http://www.denatran.gov.br/frota.htm> acessado em: 06 Jun. 2014;

Fernando P. Automação de sistemas \& robótica, Rio de Janeiro: Ed. AXCEL BOOKS, 2002. p.[13]-14.

PSA D. 2012. Eco Parking. São Paulo, v.1, n.1, p. 5-9, 2013;

\section{AUTOMATED PARKING PROTOTYPE USING MATRIX COMPUTER MODEL AND ARDUINO MICROCONTROLLER}

\author{
Thayanne Barros Bandeira, thayane.bandeira@ hotmail.com ${ }^{1}$ \\ Wellington Fonseca, Fonseca@ ufpa.br ${ }^{2}$ \\ Filipe Cavalcanti Fernandes, 13filipe11@gmail.com ${ }^{3}$ \\ Alberto Chéquer Novaes Neto, alberto.chequer@gmail.com ${ }^{4}$
}


${ }^{1}$ Universidade Federal do Pará - Campus Universitário de Tucuruí - Faculdade de Engenharia Elétrica - Rodovia BR 22 Km 13 - Canteiro de Obras - UHE Tucuruí - Vila Permanente 68464-000 - Tucuruí - Pará

${ }^{2}$ Universidade Federal do Pará - Campus Universitário de Tucuruí - Faculdade de Engenharia Elétrica - Rodovia BR 22 Km 13 - Canteiro de Obras - UHE Tucuruí - Vila Permanente 68464-000 - Tucuruí - Pará

${ }^{3}$ Universidade Federal do Pará - Campus Universitário de Tucuruí - Faculdade de Engenharia Elétrica - Rodovia BR 22 Km 13 - Canteiro de Obras - UHE Tucuruí - Vila Permanente 68464-000 - Tucuruí - Pará

${ }^{4}$ Universidade Federal do Pará - Campus Universitário de Tucuruí - Faculdade de Engenharia da ComputaçãoRodovia BR 22 Km 13 - Canteiro de Obras - UHE Tucuruí - Vila Permanente 68464-000 - Tucuruí - Pará

\begin{abstract}
A gift in daily population of large cities reality is the increase in the vehicle fleet, which every day becomes more expressive as we grow these large urban centers and the auto market is stimulated. This dilemma is directly related to the difficulty of motorists to find available parking spaces, both public and private. As a result, private foreign companies look to implement complex systems and expensive automation and control in parking management. Bearing in mind this approach, this paper proposes the development of a control system integrating concepts in electronics and computing, able to operate and manage through parking lots of medium and large automated procedures. This system has its operating principle the matrix computational model. The project uses the open source Arduino platform to locate and count the vacancies available through ultrasonic sensors that detect the presence of the vehicle. Following through a communication system where the vacancy sharing the same connection and each is identified by a binary number, the microcontroller is responsible to send, to displays located along the trajectory of the vehicle, the direction and the total number of vacancies unoccupied, referring to the general public as to the elderly and people with special needs. However, the aim of this work is to develop an effective method for movement of cars within these establishments especially in times of great flux.
\end{abstract}

Keywords: Parking, Automation, Arduino. 